\title{
Photostimulable Phosphor Glass for Ionizing Radiation Monitoring ${ }^{\dagger}$
}

\author{
Hidehito Nanto ${ }^{1,2, *}$, Kazuki Hirasawa ${ }^{1}$, Yoshinori Takei ${ }^{1}$, Yuka Yanagida ${ }^{2}$, Yoshihiro Koguchi ${ }^{2}$ \\ and Takayoshi Yamamoto ${ }^{2}$
}

1 Advanced. Materials. Science. R\&D Center, Kanazawa Institute of Technology, 3-1 Yatsukaho, Hakusan 924-0838, Japan; k.hirasawa@neptune.kanazawa-it.ac.jp (K.H.); takei@neptune.kanazawa-it.ac.jp (Y.T.)

2 Oarai Research Center, Chiyoda Technol Co., 3681 Narita, Oarai, Ibaraki 311-1313, Japan; miyamoto-y@c-technol.co.jp (Y.Y.); Koguchi-y@c-technol.co.jp (Y.K.); ecrebplsm@incloud.com (T.Y.)

* Correspondence: hnanto@neptune.kanazawa-it.ac.jp; Tel.: +81-76-274-9261

† Presented at the Eurosensors 2018 Conference, Graz, Austria, 9-12 September 2018.

Published: 12 December 2018

\begin{abstract}
Silver ( $\left.\mathrm{Ag}^{+}\right)$doped phosphate glass after exposure to ionizing radiation has an intense luminescence by stimulating with ultra-violet light. This phenomenon is called radiophotoluminescence (RPL). The RPL intensity was increased linearly with increasing $X$-ray irradiation dose up to about $1000 \mathrm{~Gy}$. Small amount of bead-type RPL glass as large as about $0.05 \mathrm{~mm}$ was well prepared with a jet flame system. It was confirmed that the RPL glass beads could be used as passive-type radiation dosimeters. Visualization and monitoring system of radiation dose distribution utilizing RPL phenomenon in the $\mathrm{Ag}^{+}$-doped phosphate glass are developed in this study. In the presentation, the basic luminescence properties are reported as well as the application of $\mathrm{Ag}^{+}$-doped phosphate glass beads to radiation dose distribution monitoring.
\end{abstract}

Keywords: phosphate glass; radiophotoluminescence; ionizing radiation monitoring; radiation dose distribution

\section{Introduction}

Silver $\left(\mathrm{Ag}^{+}\right)$doped phosphate glass irradiated with ionizing radiation exhibit an intense emission of visible photons when excited by ultraviolet (UV) light. This phenomenon is called radiophotoluminescence (RPL). The RPL phenomenon in $\mathrm{Ag}^{+}$-doped phosphate glass has, therefore, been applied to a passive-type radiation dosimeter. When the glasses are exposed to ionizing radiation, electron-hole pairs will be induced in the glass. The electrons are captured into Ag+ ions in the glass structure and then the $\mathrm{Ag}^{+}$ions change to $\mathrm{Ag}^{0}$ ions. On the other hand, the holes are captured by $\mathrm{PO} 4$ tetrahedra at the beginning of the migration and then the holes come to produce the $\mathrm{Ag}^{2+}$ ions due to interaction with $\mathrm{Ag}^{+}$ions over time. It has been reported that both $\mathrm{Ag}^{0}$ and $\mathrm{Ag}^{2+}$ ions can be luminescence centers in the glass [1-3]. The RPL intensity is proportional to the amount of ionizing radiation, meaning that RPL in the glass can be utilized as passive-type dosimeters. The RPL glass dosimeter has excellent characteristics such as honmogeneous and stable radiation sensitivity, negligible fading and reliable repeatability in radiation dose measurement. The RPL luminescence centers never disappear after reading out the accumulated information. Therefore, glass dosimeter has been widely used as passive type personal dosimeter at many radiation facilities. We have tried to develop new bead- and sheet-type glass dosimeters that are usable from low to high dose conditions such as nuclear emergencies, especially for radiation dose monitoring near or inside the Fukushima Nuclear Power Plant-1 (NPP-1) in which a large amount and many kind of radioactive materials were released by a catastrophic earthquake on 11 March 2011. In this paper, two-types of glass dosimeter, 
the bead-type and the sheet-type, are developed to adapt to various environments in radioactively contaminated areas. The sheet-type glass dosimeter with a two-dimensional RPL reader is applied to monitoring and visualization of radiation dose distribution.

\section{Results and Discussion}

Figure 1 shows typical optical absorption spectrum (left figure) and RPL emission spectrum (right figure) of $\mathrm{X}$-ray irradiated $\mathrm{Ag}^{+}$-doped phosphate glass using a fluorescence spectrometer (Hitachi F-2000). The inset of the left figure shows colored glass due to absorption in the visible wavelength. The inset in the right figure shows RPL emission image when glass was excited with 365 UV light. It is seen from Figure 1 that there are two RPL emission bands such as blue emission peaked at about $460 \mathrm{~nm}$ and an orange emission peaked at about $560 \mathrm{~nm}$. It was confirmed that the RPL intensity increased with X-ray absorbed dose. Figure 2 shows RPL image as a function of X-ray absorbed dose. To prepare a novel passive-type dosimeter system, which can be applied to radiation dose monitoring and visualization of the two-dimensional distribution of ionizing radiation in a contaminated area, we tried to develop bead- and sheet-type glass dosimeter utilizing RPL phenomenon in $\mathrm{Ag}^{+}$-doped phosphate glass. We prepared beads of $\mathrm{Ag}^{+}$-doped phosphate glass using jet-flame method as shown in Figure 3. Figure 4 show RPL emission image of prepared bead- type glass dosimeter with a diameter of about $0.05 \mathrm{~mm}$, indicating that a homogeneous RPL emission was observed.

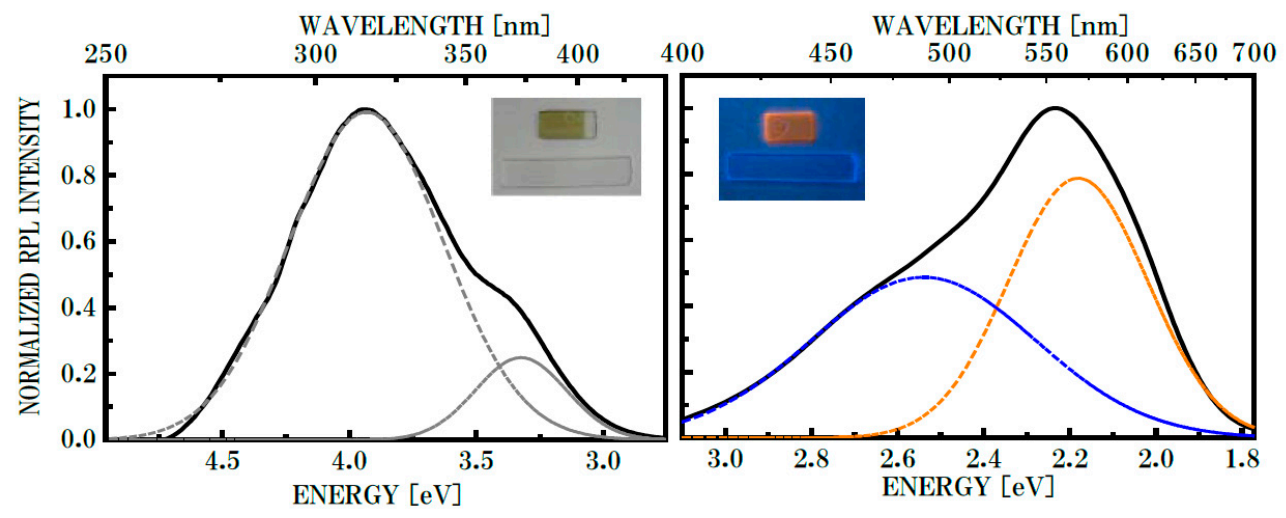

Figure 1. Typical RPL excitation (left) and emission (right) spectra of X-ray-irradiated $\mathrm{Ag}^{+}$-doped phosphate glass using a fluorescent spectrometer (Hitachi F-2010). The inset of the left figure shows dark colored glass due to absorption band in visible wavelength and the right shows RPL emission when the glass was excited with $365 \mathrm{~nm}$ light.

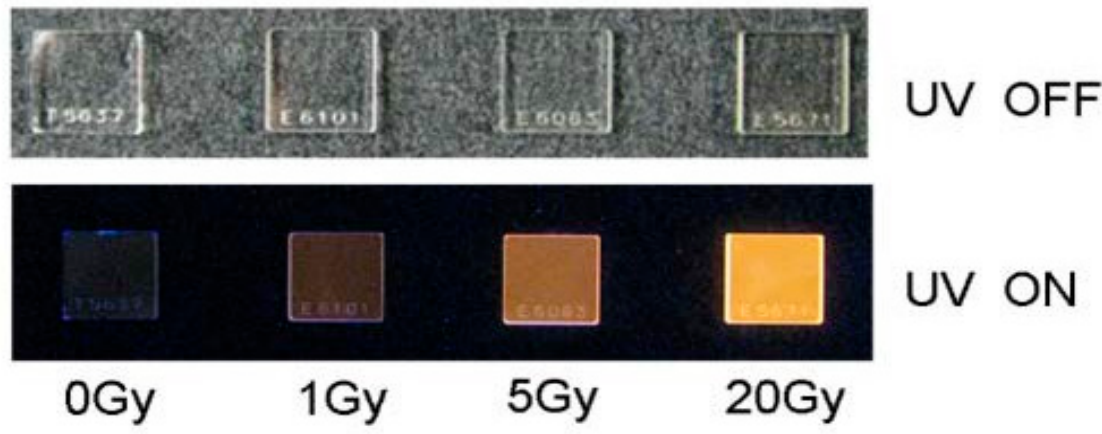

Figure 2. RPL emission image of $\mathrm{Ag}^{+}$-doped phosphate glass as a function of $\mathrm{X}$-ray absorbed dose. The upper shows UV excitation off and lower UV excitation on. 


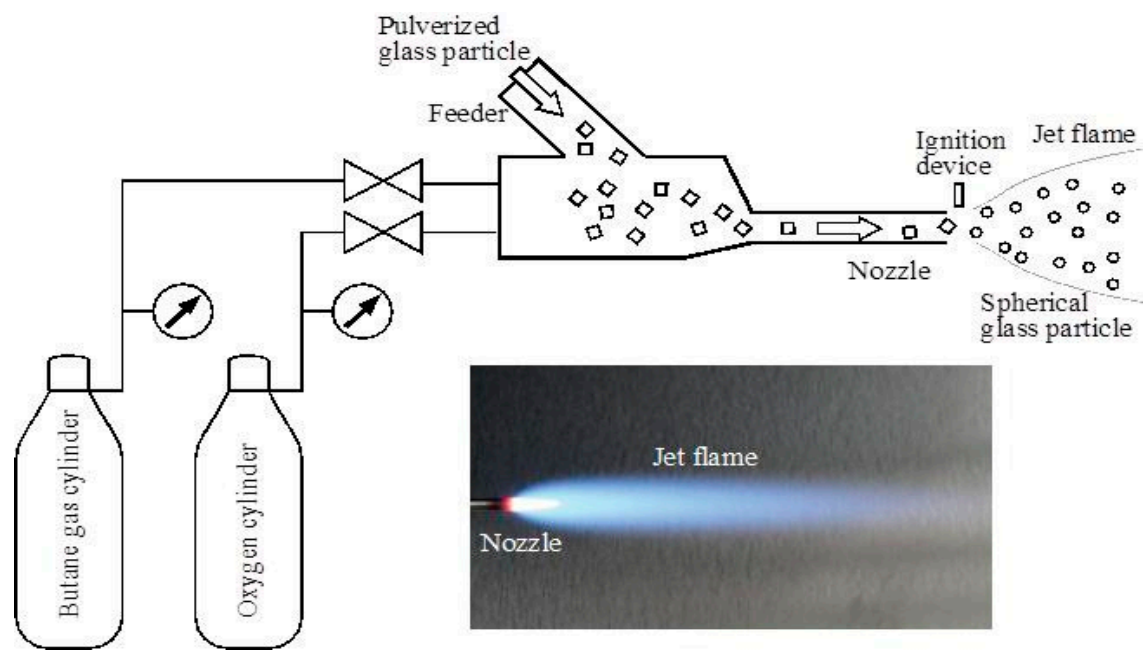

Figure 3. Process of prepared bead-type RPL glass dosimeter using Jet-frame method.

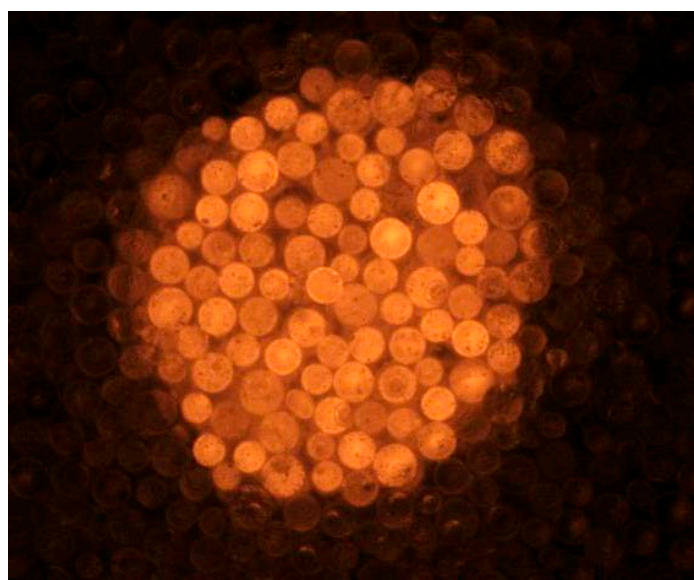

Figure 4. RPL emission image of bead-type $\mathrm{Ag}^{+}$-doped phosphate glass.

The sheet-type RPL glass dosimeter was made by kneading the mixture of bead-type glass with homogeneous diameter and an acrylic binder on an acrylic resin film. It was confirmed that the acrylic resin film excited with UV light did not exhibits luminescence. Figure 5 shows the two dimensional dose distribution observed from the sheet-type glass dosimeter, which was uniformly irradiated with gamma-rays. Figure 6 shows RPL intensity of sheet-type glass dosimeter as a function of absorbed dose of gamma-rays. It can be seen from Figure 6 that the RPL intensity is increased linearly with absorbed dose, indicating that the sheet-type glass dosimeter can be used for the evaluation and visualization of radiation dose distribution.

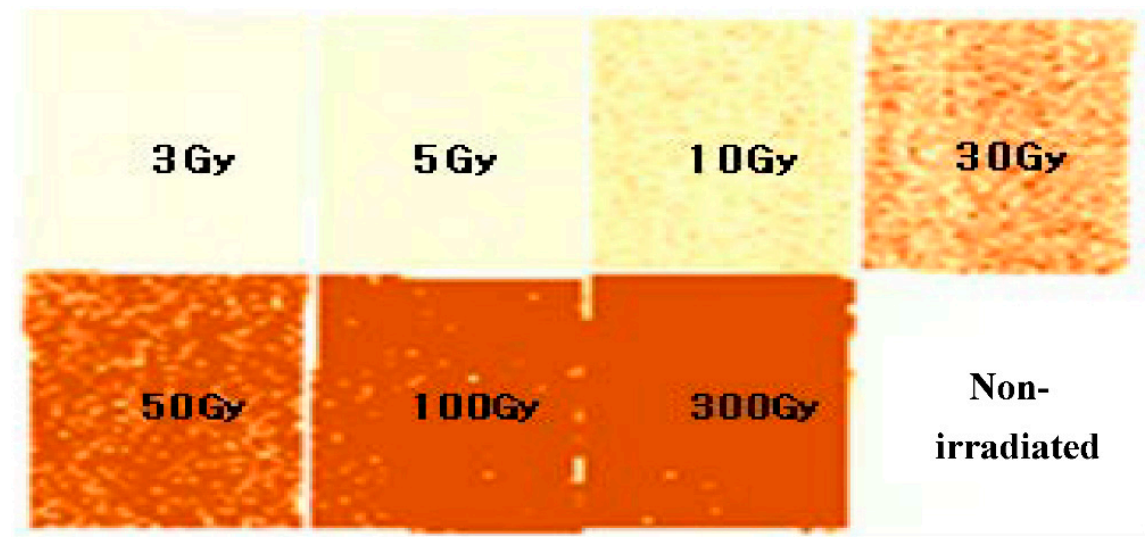

Figure 5. RPL emission image of sheet-type $\mathrm{Ag}^{+}$-doped phosphate glass which is irradiated with homogeneous gamma-rays in the range from 3 [Gy] to 300 [Gy]. 


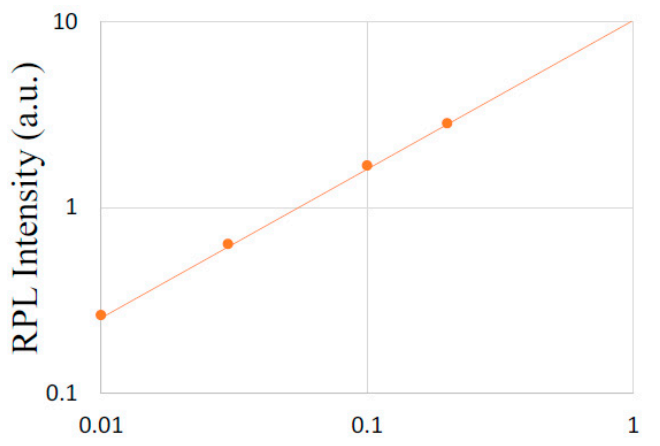

Absorbed dose (Gy)

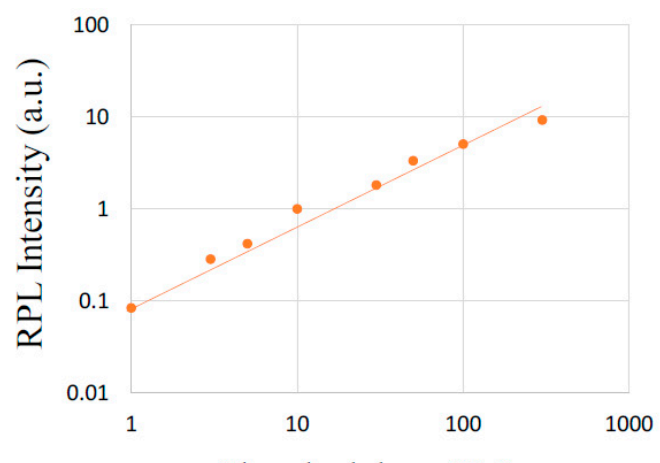

Absorbed dose (Gy)

Figure 6. RPL intensity of sheet-type glass dosimeter as a function of gamma-rays absorbed dose in the range from $0.01[\mathrm{~Gy}]$ to 300 [Gy].

The flexible RPL glass sheet prepared is expected to be useful for two-dimensional radiation imaging. The research on radiation monitoring using the bead-type and the sheet-type glass dosimeters in contaminated area of Fukushima NPP-1 as shown in Figure 7 is in progress.

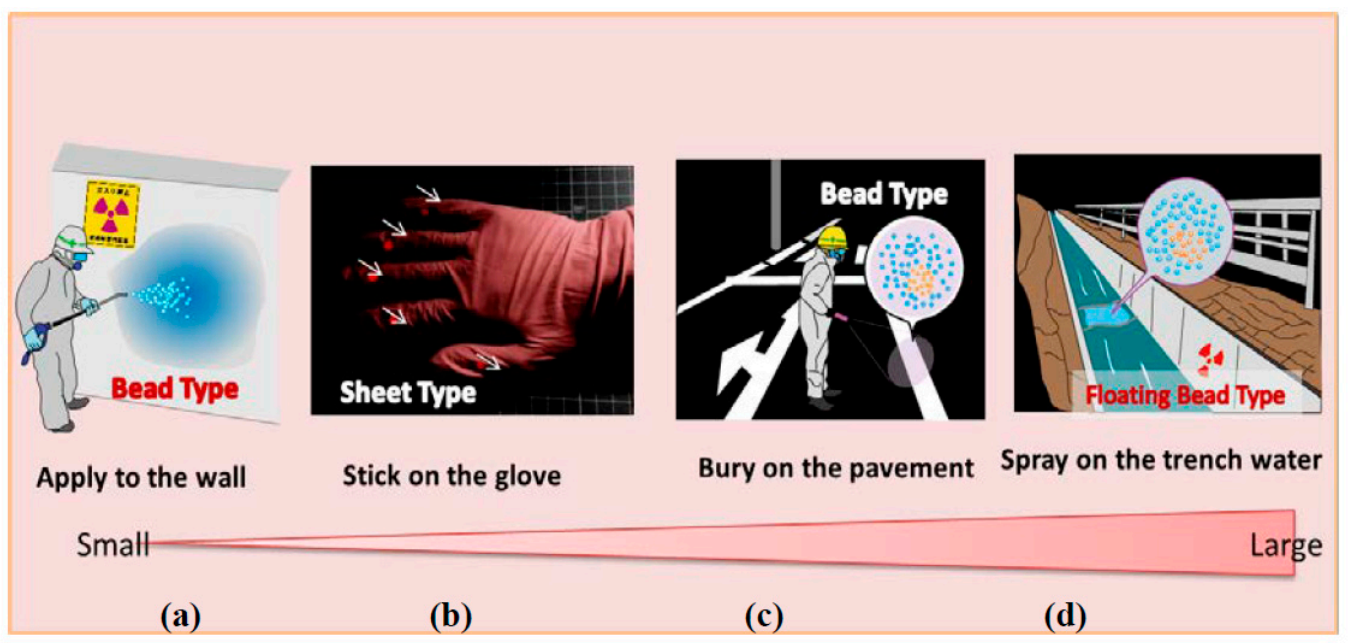

Figure 7. A schematic diagram for applications of the bead-type and sheet-type glass dosimeter in the cases such as (a) apply to wall, (b) stick on the glove, (c) bury on the pavement and (d) spray on the trench water.

Acknowledgments: The authors wish to express their gratitude to T. Kurobori of Kanazawa University for his fruitful discussion on RPL spectrum measurement. This study was partly supported by a Grant-in-Aid for Challenging Exploratory Research (15K13399).

\section{References}

1. Miyamoto, Y.; Kinoshita, K.; Koyama, S.; Takei, Y.; Nanto, H.; Yamamoto, T.; Sakakura, M.; Shimotsuma, Y.; Miura, K.; Hirao, K. Emission and excitation mechanism of radiophotoluminescence in $\mathrm{Ag}^{+}$-dactivated phosphate glass. Nuclear Instrum. Methods 2010, A619,71-74.

2. Kurobori, T.; Wang, Z.; Miyamoto, Y.; Nanto, H.; Yamamoto, T. The role of silver in the radiophotoluminescent properties in silver-activated phosphate glass and sodium chloride crystal. Opt. Mater. 2010, 32, 1231-1236.

3. Nanto, H. Photostimulable strage phosphor materials and their application to radiation monitoring. Sens. Mater. 2018, 30, 327-337.

(C) 2018 by the authors. Licensee MDPI, Basel, Switzerland. This article is an open access article distributed under the terms and conditions of the Creative Commons Attribution (CC BY) license (http://creativecommons.org/licenses/by/4.0/). 